\title{
Nanocrystalline Particles of CoCrFeMnMoNi High Entropy Alloy as Reinforcement Material in an Al Matrix
}

\author{
M. F. Bujanda-Sánchez ${ }^{1}$, I. Estrada-Guel ${ }^{2}$, R. Martinez-Sánchez ${ }^{2}$, A. Torres-Garcia ${ }^{1}$, C. A. Rodríguez- \\ González $^{3}$, A. Duarte-Moller², C. D. Gómez-Esparza² \\ ${ }^{1}$ Universidad Tecnológica de Ciudad Juárez, Av. Universidad Tecnológica No. 3051, Col. Lote Bravo II, \\ 32695, Cd. Juárez, Chih., Mexico. \\ ${ }^{2}$ Centro de Investigación en Materiales Avanzados (CIMAV), Laboratorio Nacional de \\ Nanotecnología, Miguel de Cervantes No. 120, 31136, Chihuahua, Chihuahua, Mexico. \\ ${ }^{3}$ Instituto de Ingeniería y Tecnología, Universidad Autónoma de Ciudad Juárez (UACJ), Av. Del Charro \\ 450 norte, 32310, Cd. Juárez, Chih., Mexico.
}

Among the advanced structural materials for applications in the aerospace, defense and automotive industries, the aluminum-based metal matrix composites have increased their demand due to their attractive mechanical properties such as their high modulus of elasticity and high mechanical strength [1]. The ceramic particles have been preferentially selected to reinforce metallic matrices due to their high rigidity, strength and hardness. However, there are some limitations for the preparation of these composite materials, such as the low wettability and chemical compatibility between the ceramic particles and the metal matrix. To overcome these limitations, Madhu et al. [2] proposed some "metalmetal composites", choosing conventional alloy systems with restricted solubility between them. In recent years, aluminum-based matrix composites reinforced with metal particles have been studied as potential alternatives to reduce the weight of components for structural applications and to improve their mechanical and physical properties.

High entropy alloys (HEA) are a new class of high performance alloys, developed in 2004 by Yeh et al. [3]. With at least 5 main elements in equiatomic or close to equiatomic composition, these alloys possess excellent properties such as: high mechanical strength, hardness, ductility and thermal stability at high temperatures, as well as high resistance to wear and oxidation. Through the mechanical alloying technique for the production of HEA, a wide variety of nanocrystalline particles can be generated to be used as reinforcements for metal-metal composite materials fabrication. There are few publications implying the use of HEA as reinforcement of metal matrices, specifically for aluminum based composites [4]. The present investigation deals with the synthesis of aluminum-based composites reinforced by the dispersion of HEA nanocrystalline particles, as well as the microstructural evolution study of the composites and their mechanical response.

A nanocrystalline CoCrFeMnMoNi HEA was synthesized by mechanical alloying in a high-energy SPEX 8000M mill for 10h under an inert Ar atmosphere. The HEA particles were dispersed into an Al matrix in amounts of 1, 5, 10 and $20 \mathrm{wt} . \%$ by mechanical milling (2h). Solid samples were obtained by cold-pressing at $900 \mathrm{MPa}$ and sintering at $500^{\circ} \mathrm{C}$ for $3 \mathrm{~h}$, under Ar atmosphere. The composites were evaluated by scanning electron microscopy (SEM), X-ray diffractometry (XRD) and microhardness testing.

Through SEM analysis (Fig. 1), is it possible to observe that mechanical milling was an efficient method to obtain a homogeneous dispersion of HEA particles into the aluminum matrix. After sintering, the microstructure of composites shows a uniform dispersion of HEA particles. Surface defects such as 
cracks and pores decrease with the increase of HEA content (Figs. 2a-d). In the XRD patterns of sintered composites (Fig. 2d), only the diffraction Bragg peaks of Al are present and there is no evidence about the formation of a new phase; due to CoCrFeMnMoNi particles exhibit stability as solute in the aluminum matrix. Microhardness results show that composites hardness increases as a function of HEA content from 99 to $234 \mathrm{HV}$ for composites reinforced with 1 and $20 \mathrm{wt}$.\%, respectively.

\section{References:}

[1] S T Mavhungu, E T Akinlabi, M A Onitiri and F M Varachia, Procedia Manuf., 7 (2017) p. 178.

[2] S Madhusudan, M M M Sarkar and N R M R Bhargava, Int. J. Alloys Compd. 47 (2009) p. 116.

[3] J W Yeh, S K Chen, S J Lin, J Y Gan, T S Chin, T T Shun, C H Tsau, S Y Chang, Adv. Eng. Mater. 6 (2004) p. 299.

[4] K Praveen Kumar, M Gopi Krishna, J Babu Rao, N R M R Bhargava, J. Alloys Compd. 640 (2015) p. 421.
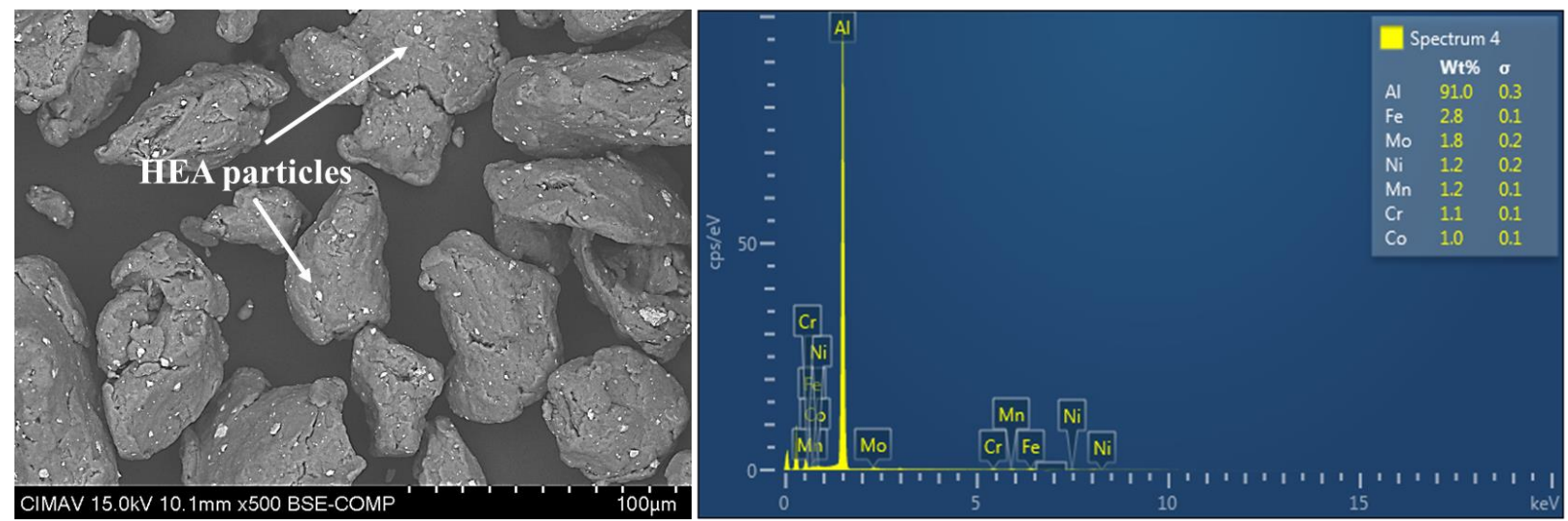

Figure 1. BSE-SEM micrograph of aluminum powder (gray particles) after dispersion of 10 wt. $\%$ of $\mathrm{CoCrFeMnMoNi}$ particles (bright particles, heavy elements) by mechanical milling. The EDS results give us some evidence about the chemical composition of composites is very close to the calculated compositions.
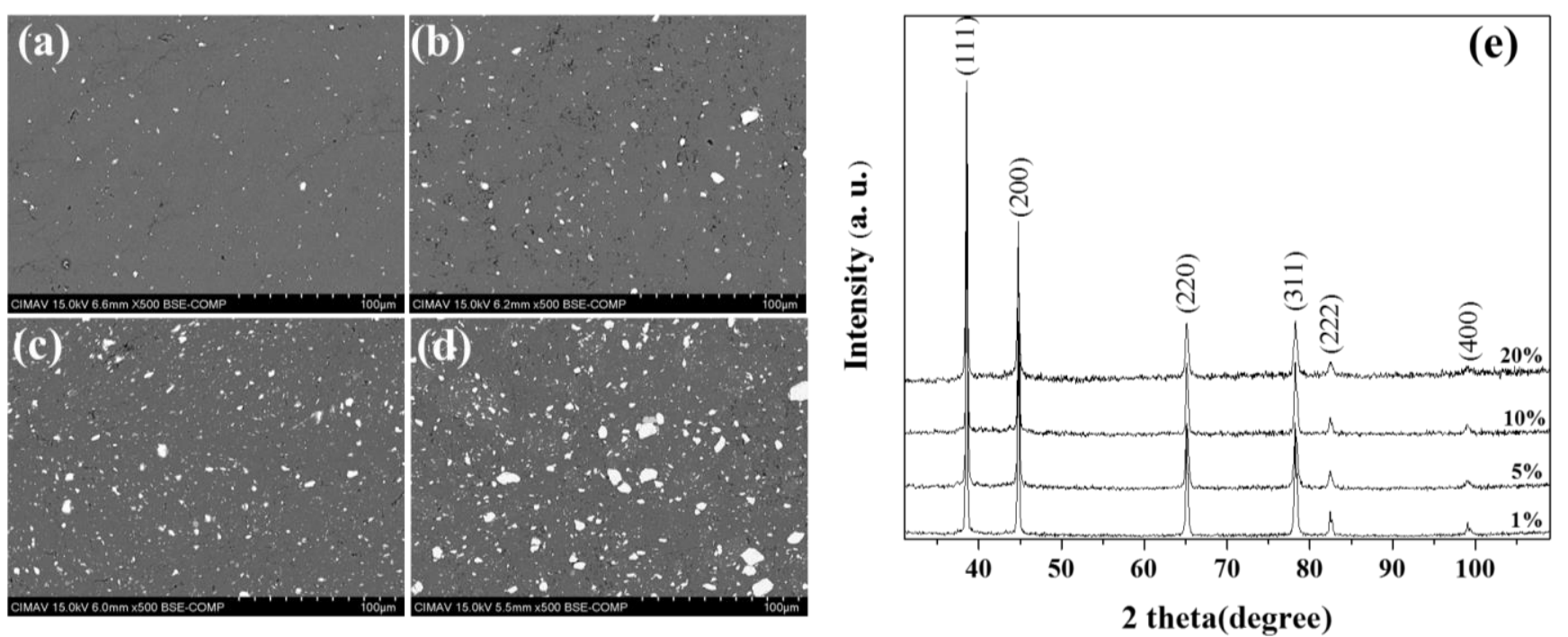

Figure 2. (a) FESEM-SE micrographs of sintered Al-based composites reinforced with: a) 1, b) 5 , c) 10 and d) 20 wt. \% of nanocrystalline CoCrFeMnMoNi powder particles, and e) X-ray diffraction patterns of aluminum composites as a function of HEA particles content. 